Please do not remove this page

RMIT

UNIVERSITY

\title{
Terahertz localized surface plasmon resonances in coaxial microcavities
}

Withayachumnankul, Withawat; Shah, Charan Manish; Fumeaux, Christophe; Kaltenecker, K; Walther, Markus; Fischer, B; Abbott, Derek

https://researchrepository.rmit.edu.au/esploro/outputs/9921861946501341/filesAndLinks?institution=61RMIT_INST\&index=null

Withayachumnankul, W., Shah, C. M., Fumeaux, C., Kaltenecker, K., Walther, M., Fischer, B., Abbott, D., Bhaskaran, M., \& Sriram, S. (2013). Terahertz localized surface plasmon resonances in coaxial microcavities. Advanced Optical Materials, 1(6), 443-448. https://doi.org/10.1002/adom.201300021 Document Version: Accepted Manuscript

Published Version: https://doi.org/10.1002/adom.201300021

Repository homepage: https://researchrepository.rmit.edu.au

(c) 2013 WILEY-VCH Verlag GmbH \& Co. KGaA, Weinheim

Downloaded On 2023/04/26 16:28:36 +1000

Please do not remove this page 
Thank you for downloading this document from the RMIT Research Repository.

The RMIT Research Repository is an open access database showcasing the research outputs of RMIT University researchers.

RMIT Research Repository: http://researchbank.rmit.edu.au/

\section{Citation:}

Withayachumnankul, W, Shah, C, Fumeaux, C, Kaltenecker, K, Walther, M, Fischer, B, Abbott, D, Bhaskaran, M and Sriram, S 2013, 'Terahertz localized surface plasmon resonances in coaxial microcavities', Advanced Optical Materials, vol. 1, no. 6, pp. 443-448.

See this record in the RMIT Research Repository at:

http://researchbank.rmit.edu.au/view/rmit:21350

Version: Accepted Manuscript

Copyright Statement: (c) 2013 WILEY-VCH Verlag GmbH \& Co. KGaA, Weinheim

Link to Published Version:

http://dx.doi.org/10.1002/adom.201300021 
DOI: 10.1002/adom.201300021

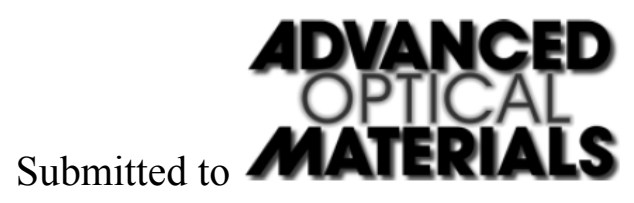

Terahertz Localized Surface Plasmon Resonances in Coaxial Microcavities

By Withawat Withayachumnankul,* Charan Manish Shah, Christophe Fumeaux, Korbinian Kaltenecker, Markus Walther, Bernd M. Fischer, Derek Abbott, Madhu Bhaskaran, and Sharath Sriram*

W.W. dedicates this work to the memory of Boonchai Withayachumnankul.

[*] Dr. Withawat Withayachumnankul, Prof. Christophe Fumeaux, Dr. Bernd M. Fischer, Prof. Derek Abbott

School of Electrical \& Electronic Engineering

The University of Adelaide, Adelaide, South Australia 5005 (Australia)

E-mail: withawat@eleceng.adelaide.edu.au

[*] Dr. Withawat Withayachumnankul, Mr. Charan Manish Shah, Dr. Madhu Bhaskaran, Dr. Sharath Sriram

Functional Materials and Microsystems Research Group

RMIT University, Melbourne, Victoria 3001 (Australia)

E-mail: sharath.sriram@gmail.com

Mr. Korbinian Kaltenecker, Dr. Markus Walther

Department of Molecular and Optical Physics

Albert-Ludwigs-Universität Freiburg, 79104 Freiburg (Germany)

Mr. Korbinian Kaltenecker, Dr. Bernd M. Fischer

French-German Research Institute Saint-Louis

5 rue du Général Cassagnou, 68301 Saint-Louis Cedex (France)

Keywords: plasmonics, localized surface plasmon resonance, terahertz, metamaterial, coaxial cavity

Coaxial microcavities etched into the surface of a doped silicon substrate are shown to support localized surface plasmon resonances (LSPRs) at terahertz frequencies. The underlying mechanism involves coupling of freely propagating terahertz waves with surface plasmon polaritons (SPPs), which propagate in a coaxial mode along the cavity walls in the axial direction. A Fabry-Pérot resonance is built up when the SPP wavenumber appropriately relates to the cavity depth. Owing to the Ohmic loss of the silicon at terahertz frequencies, the energy of the resonating SPPs is largely dissipated, leading to a modified reflection spectrum.

Strong field enhancement is observed inside the cavities at resonance. The theoretical analysis 


\section{ADVANCED \\ Submitted to \\ MATERIALS}

is supported by numerical and experimental results. This study is a promising pathway for development of terahertz devices with applications in the areas of photonic integrated circuits, molecular sensing, and subwavelength imaging.

\section{Introduction}

The last twenty years have seen significant progress in terahertz research, driven by the introduction of coherent sources and detectors such as photoconductive antennas, electrooptic crystals, and quantum cascade lasers. In recent years, terahertz technology has seen another large leap with the advent of solid-state terahertz sources operating at room temperature. ${ }^{[1-3]}$ This latest development may potentially propel some fundamental concepts, including terahertz sensing, imaging, and communications, towards commercial products. Along the path of terahertz technology development, increasing the wave confinement beyond the diffraction limit is a major challenge with significant implications. Enhanced wave confinement promises increased spatial resolution that could potentially lead to singlemolecule sensing in the terahertz region, where numerous substances exhibit rich spectroscopic features.${ }^{[4]}$ Integrated optics essential for terahertz communication applications is another area that could benefit from this breakthrough through component miniaturization. This challenge is extremely relevant due to the relatively long wavelengths of terahertz waves. The spatial confinement of a propagating wave is limited by diffraction, and is thus a function of the wavelength. This limitation can be circumvented by using surface plasmon polaritons $(\mathrm{SPP}){ }^{[5]}$ the bound electron oscillations propagating along the interface between a dielectric and a conductor. For Drude conductors, SPPs can be sustained at frequencies right below the plasma frequency, where the real permittivity is slightly negative and the imaginary part is small. This region lies in the visible and UV ranges for most metals. At terahertz frequencies, the properties of metals approach a perfect electric conductor (PEC), leading to field 


\section{Submitted to

delocalization into grazing waves or Sommerfeld-Zenneck waves. ${ }^{[6-8]}$ Periodically-structured metal surfaces can help to increase the confinement of surface waves at these frequencies to achieve so-called designer or spoof SPPs. ${ }^{[9-12]}$ Additionally, moderately-doped semiconductors with a plasma frequency dependent on the carrier concentration are shown to support terahertz SPPs. ${ }^{[13-16]}$

A localized surface plasmon resonance (LSPR) is a manifestation of surface plasmons that can exist in subwavelength conductive elements. Typically, the resonance takes place at equilibrium between the charge displacement force, via an external excitation, and the ionic restoration force. The size and shape of elements, together with the properties of materials, determine the resonance characteristics. Recently, a few different types of elements made of semiconductors have been shown to support LSPRs at terahertz frequencies, including dipoles, ${ }^{[17]}$ bowties, ${ }^{[17,18]}$ and coupled disks. ${ }^{[19]}$ Owing to strong field localization in a subwavelength volume, terahertz LSPRs are very attractive for many applications, including sensing and imaging. ${ }^{[20]}$ As an alternative to resonant plasmonic elements, complementary elements in the form of resonant cavities have a potential to sustain terahertz LSPRs, but have yet to be realized. Nonradiative modes of these resonant cavities promise minimal inter-cell coupling, whilst the self-supporting structure of the cavities reduces the fabrication complexity.

In this article, a form of LSPR cavities, namely coaxial microcavities, is investigated and realized at terahertz frequencies. A single coaxial microcavity can be described as a blind annular hole etched into a doped semiconductor wafer, which intrinsically supports terahertz SPPs. The resonance mechanism is closely related to that of optical coaxial (or annular) aperture arrays, which exhibit extraordinary transmission via the interplay between cylindrical SPPs and cavity resonance modes. ${ }^{[21-25]}$ Another related structure is an array of coaxial nanocavities with a V-shaped groove, which has been demonstrated recently at optical 


\section{Submitted to

frequencies on metallic surfaces. ${ }^{[26,27]}$ As opposed to the straight profile, the tapered profile of the groove gives rise to the depth dependence of the SPP characteristics. These optical apertures and cavities form a basis for the study of straight-profile coaxial microcavities sustaining LSPRs at terahertz frequencies. It is worth noting that the principle of these optical and terahertz coaxial cavities is radically different from the realization of terahertz blind annular holes on a copper surface. ${ }^{[1,12]}$ In these studies, terahertz spoof SPPs were established over the metallic surface, which was textured to realize an effective plasma frequency. Those holes were operated below the cutoff to allow evanescent fields perpendicular to the apertures, with no surface plasmons and LSPRs in the holes.

\section{Design, Fabrication, and Experiments}

A unit cell of the coaxial microcavity is illustrated in Figure 1, along with the dimensions and the incident field vectors. The cavity is described as an annular air-filled groove with a straight cross-sectional profile that is etched into a homogeneous material, in this case a moderately doped silicon substrate. Each sample is composed of thousands of identical cavities, periodically arranged as a 2D array on the substrate. As shown in Table 1, samples with two different cavity depths are investigated to observe variations of the plasmon resonance. The average radius of the annular groove is fixed for all of the designs, resulting in a nearly constant cutoff frequency of the resonance mode. The various wall separations introduce different conditions for the mode confinement. The wafer is thick enough to suppress field transmission to the other side. The choice of silicon as a substrate is based on availability and manufacturability, although InSb has a higher carrier mobility or equivalently lower dissipation. It will be demonstrated later that doped silicon can reasonably support nonpropagating terahertz LSPRs, despite a moderate electron mobility and hence a moderate plasmonic absorption. 
Submitted to

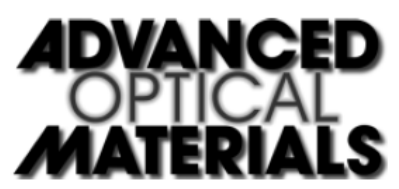

According to the specification, the silicon wafers are $\langle 100\rangle$ oriented and n-type doped with phosphorus. The material resistivity lies in between $0.02-0.05 \Omega \mathrm{cm}$. In order to experimentally determine the optical properties of these wafers at terahertz frequencies, the measured complex reflectivity of the bare silicon wafer is fitted with the Fresnel equation incorporating the Drude model. ${ }^{[28]}$ In this procedure, the carrier concentration is the only free parameter, whilst the electron mobility is determined from this concentration through an empirical model. ${ }^{[29]}$ As a result, the carrier concentration of the silicon at room temperature is estimated as $2.0 \times 10^{17} \mathrm{~cm}^{-3}$, yielding a plasma frequency of $7.87 \mathrm{THz}$, whilst the electron mobility is estimated as $603 \mathrm{~cm}^{2} \mathrm{~V}^{-1} \mathrm{~s}^{-1}$, and hence the collision frequency equals $1.78 \mathrm{THz}$. The DC resistivity estimated from the mobility and the concentration equals $0.05 \Omega \mathrm{cm}$, within the range specified by the manufacturer. From these quantities, the complex permittivity at $1.0 \mathrm{THz}$ is determined to be $-3.14-26.45 j$. Hence, the SPP propagation length on the silicon-air interface is $1.27 \mathrm{~mm}$, and the decay lengths normal to the interface are $246 \mu \mathrm{m}$ into the air and $9.24 \mu \mathrm{m}$ into the silicon substrate.

The coaxial microcavities are fabricated by using conventional micro-fabrication techniques involving photolithography and deep reactive ion etching (DRIE). The fabricated structures are then examined using a Philips XL-30 scanning electron microscope (SEM) to determine the depth and smoothness of the vertical walls obtained from the DRIE process. Figure 2 shows the SEM micrographs of the coaxial microcavities imaged with a $45^{\circ}$ tilt. The SEM micrographs confirm that smooth and straight sidewalls are obtained. One of the wafers is cleaved and loaded orthogonally into the SEM to image the cross-sectional depth of the etched wafers (not shown here), which confirms the data obtained from a profilometric analysis. The total area for each sample is $10 \times 10 \mathrm{~mm}^{2}$, containing about 2500 identical cavities. To provide a reference in the measurements, the surface of a bare silicon wafer is coated with a $200 \mathrm{~nm}$ thick gold layer to create a terahertz mirror. 


\section{Submitted to

The measurement is carried out in reflection-mode terahertz time-domain spectroscopy (THz-TDS) with a pair of emitting/detecting photoconductive antennas (PCAs) on LT-GaAs substrates. The generated linearly polarized terahertz beam propagates through a beam splitter and is then focused by an elliptical mirror to a focal beam diameter of $8 \mathrm{~mm}$ onto the sample at normal incidence. The beam reflected from the sample is collected by the same mirror back to the beam splitter that partially reflects the energy into the PCA detector. The entire terahertz path is purged with nitrogen to avoid water vapor absorption lines. The total scanning duration is $68 \mathrm{ps}$ and is digitized with a step size of $0.06 \mathrm{ps}$. For comparison, electromagnetic simulations are performed with a commercial full-wave frequency-domain solver, CST Microwave Studio, based on the finite-element method. In the simulated models, the dielectric properties of the silicon are described via the Drude model with the plasma frequency and scattering rate estimated earlier. Unit-cell boundary conditions are employed for the transverse boundaries to replicate an infinite planar array. A Floquet port excites the array with a normally incident plane wave and also collects the scattered field. The simulation using ANSYS HFSS with a similar setting produces comparable results.

\section{Design, Fabrication, and Experiments}

The normalized reflection magnitude profiles for the $50 \mu \mathrm{m}$ depth cavity arrays are shown in

Figure 3. The measurement and simulation results are in general agreement with slight discrepancies due to fabrication tolerances, system noise, and sample alignment. No transmission through the wafer is detectable within the system bandwidth and dynamic range for all of the samples. The normalized reflection spectrum for the bare silicon illustrates the transition from metal to dielectric, corresponding to the zero permittivity crossing at $1.46 \mathrm{THz}$. The high reflection towards low frequencies demonstrates the metallic characteristic of the material. The response of all the microcavity samples follows the silicon reflection baseline. For all the cavities with a $50 \mu \mathrm{m}$ depth, the resonance is evident around 1.0-1.2 THz, where 


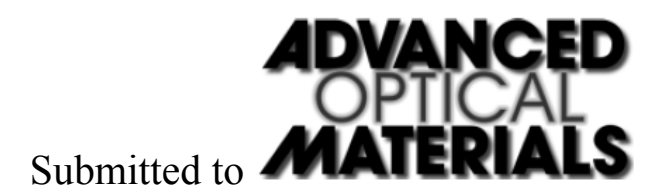

the arrays dissipate a relatively large amount of incident energy. As the wall separation in the cavities becomes narrower, the resonance frequency undergoes redshift, and the resonance strength becomes weaker. Supplementary simulation reveals that the lattice constant has a negligible effect on the resonance characteristics, except for the resonance strength that decreases with a larger separation. Observed in the simulation results in Figure 3(b), the lattice mode is manifested as a small dip at $1.5 \mathrm{THz}$, which corresponds to the lattice constant of one wavelength. It is noteworthy that no resonance is observed in simulations of PEC cavities with the same dimensions.

The simulated field distribution on resonance is given in Figure 4 for a specific sample. The electric field pattern in Figure 4(a) resembles the TE 11 (dipolar) mode of a coaxial waveguide. The fundamental TEM mode, which is radially symmetric, cannot develop inside the cavity because of the plane wave excitation. Associated with this TE 11 mode is the strong field enhancement close to the cavity walls at the aperture, owing to the plasmonic effect and the singularity at the corners, as shown in Figures 4(b) and 5. Figure 5 further shows that a narrower wall separation leads to stronger field strength in the middle of the aperture. This developed hyperbolic field variation for the component perpendicular to the aperture walls is similar to the behavior of gap-plasmon modes exclusively found in plasmonic waveguides. ${ }^{[31]}$ In these coaxial cavity the aperture with a subwavelength gap size is excited by a nearly constant field to couple to only the even or symmetric mode. ${ }^{[32]}$ On closer inspection, the absolute electric field strength in Figure 4(b) reveals a pair of a node at the bottom and an anti-node at the top of the $50 \mu \mathrm{m}$ depth cavity. This node pair indicates the fundamental Fabry-Pérot resonance.

Further insight into the response of these cavities can be gained from the dispersion behavior of plasmonic coaxial waveguides. The nature of the SPP mode in the waveguides is close to that of the planar heterostructure comprising conductor/dielectric/conductor, where the SPP 


\section{ADVANCED \\ Submitted to \\ MATERIALS}

modes on the two interfaces interact and give rise to the coupled mode. ${ }^{[33]}$ The approximated dispersion model can be expressed in the transcendental form: ${ }^{[34]}$

$$
\tanh \left(\sqrt{\beta^{2}+\left(v / r_{\text {avg }}\right)^{2}-k_{0}^{2} \varepsilon_{\mathrm{d}}} \frac{g}{2}\right)=-\frac{\varepsilon_{\mathrm{d}} \sqrt{\beta^{2}+\left(v / r_{\mathrm{avg}}\right)^{2}-k_{0}^{2} \varepsilon_{\mathrm{d}}}}{\varepsilon_{\mathrm{c}} \sqrt{\beta^{2}+\left(v / r_{\mathrm{avg}}\right)^{2}-k_{0}^{2} \varepsilon_{\mathrm{c}}}}
$$

where $\beta$ and $k_{0}$ denote the SPP and free-space wavenumbers, respectively; $\varepsilon_{\mathrm{c}}$ and $\varepsilon_{\mathrm{d}}$ for the relative permittivities of the doped silicon and free space, respectively; $v$ for the angular momentum, equal to 1 for the TE11 mode; $r_{\text {avg }}=\left(r_{o}+r_{i}\right) / 2$; and $g=r_{o}-r_{i}$. Equation 1 yields the dispersion diagram for the designed cavities, given in Figure 6 in comparison to the dispersion relation for the PEC waveguide operating in the same mode. It is obvious that the PEC waveguide sustains fast waves, with the exact cutoff frequency at about $0.73 \mathrm{THz}$. On the other hand, the same mode in the plasmonic coaxial waveguides evolves from fast waves at low frequencies to slow waves at high frequencies. In case of the lossless plasmonic waveguides in Figure 6(a), the propagation constant goes towards infinity, as the frequency approaches the surface plasmon frequency at $1.30 \mathrm{THz}$ where $\varepsilon_{\mathrm{c}}=-1 .{ }^{[33]}$ Contrarily, the propagation constant for the lossy waveguides becomes finite at the surface plasmon frequency, as illustrated in Figure 6(b). As the wall separation is reduced, the propagation constant for the plasmonic waveguide increases, equivalent to an increase in the effective refractive index. Correspondingly, the cutoff frequency for the plasmonic waveguide becomes dependent on the wall separation.

The resonance condition has to account for the cavity length, propagation constant, and the phase changes upon reflection at both ends of the cavity. The behavior of the cavity open end is close to that of a perfect magnetic conductor (PMC) that induces a zero phase change to the reflected wave. The bottom of the cavity, made of the doped silicon, imposes about $-0.9 \pi$ reflection phase shift, estimated from a plane-wave simulation. The slight deviation from the PEC reflection phase of $-\pi$ is due to significant field penetration into the material. From this 
Submitted to

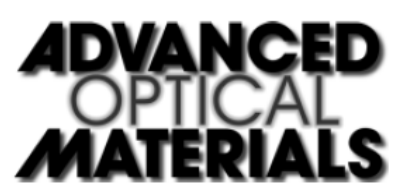

observation, the condition for the resonance can be approximately given as $\beta_{\mathrm{N}}=(2 N+m) \pi / 2 d$, where $\beta_{\mathrm{N}}$ is the SPP wavenumber inside the cavity for the $N^{\text {th }}$-order resonance $(N=0,1,2, \ldots)$, $d$ is the cavity depth, and $m=\{0.9,1.0\}$ for the plasmonic and PEC boundaries, respectively.

From Figure 6(b), the fundamental resonance $N=0$ of the $50 \mu \mathrm{m}$ depth PEC cavity operating in the TE 11 mode would take place at $1.67 \mathrm{THz}$. For the plasmonic cavities L40, L30, and L20 sustaining the same mode, the resonance frequencies are estimated to be around 1.34, 1.28, and $1.18 \mathrm{THz}$, compared with the simulation and experimental results of $1.20,1.13$, and $1.04 \mathrm{THz}$, respectively. A slight difference between the analytical and experimental results is likely from a small phase change introduced by the fringing field above the cavity opening. As the physical length of the cavity becomes shorter, in the case of a lossless plasmonic material the resonance is expected to converge to the surface plasmon frequency where the wavenumber is infinite. ${ }^{[33]}$ However, this convergence is not observed in the lossy material, since the wavenumber becomes finite. Figure 7 shows the response of the $25 \mu \mathrm{m}$ depth cavities, S20, S30, and S40 in the TE11 mode. In these cases the reflection dip can be observed around 1.5 THz. From the resonance condition and the dispersion relation, these cavities are expected to exhibit the fundamental resonance at the wavenumber of $\beta_{0}=5.7 \times 10^{4} \mathrm{~m}^{-1}$, or equivalently around $2.5 \mathrm{THz}$. However, as discussed earlier, beyond $1.46 \mathrm{THz}$ this doped silicon becomes a lossy dielectric, and hence, no longer supports the SPP mode. Therefore, the structure cannot sustain the expected resonance at $2.5 \mathrm{THz}$, and the observed dip in the reflection magnitude is in fact the resonance shoulder. On the other hand, as the physical length of the cavity becomes longer, the fundamental resonance experiences redshift, and at the same time higher-order cavity modes $N>0$ can be supported. The simulation shows that a first-order mode $(N=1)$ can be observed when the cavity depth is at least $140 \mu \mathrm{m}$. For the structure with the same radii as L30 and the depth of $140 \mu \mathrm{m}$, the fundamental and first-order 


\section{ADVANCED \\ Submitted to \\ AnIRILS}

resonances take place at $0.72 \mathrm{THz}$ and $1.35 \mathrm{THz}$, respectively. Despite that, the fabrication to achieve this level of cavity depth becomes difficult with present capabilities.

The resonance mechanism involving the plasmonic effect can be summarized as follows. A freely propagating wave is incident on the sample and then diffracted by the apertures of the subwavelength cavities. The diffraction allows phase matching and excitation of the bound mode along the cavity walls that support cylindrical SPPs. The characteristics of cylindrical SPPs depend on the mode field distribution, which in this case corresponds to a coaxial TE 11 mode, as well as the cavity geometry that influences the mode coupling between the two sidewalls. The resonance originates from the standing wave of the guided SPP inside the cavity upon reflection at its open and blind ends. In effect, the resonant cavity appears slightly longer because of the plasmonic confinement along the walls, the fringing fields at the aperture, and the field penetration at the bottom of the cavity. The observable shift in the resonance despite a fixed cavity depth is due to a reduced wall separation that results in a change in the SPP wavenumber. Resonance damping is caused by resistive loss in the silicon. Owing to the non-radiative mode in the cavity, the resonance frequency is virtually independent of the lattice constant.

\section{Conclusions}

This article has presented the concept and realization of plasmonic coaxial micro-cavities operating at terahertz frequencies. It is experimentally demonstrated that the microcavities built into a doped silicon layer can support LSPRs through the interplay between the cylindrical SPPs and the cavity modes. The interaction between the bound electromagnetic waves and surface electron oscillations effectively leads to the subwavelength dimensions of the cavity. Since the resonance originates inside the cavity, the inter-cell coupling is minimal. The dispersion model for plasmonic coaxial waveguides can be used to quantitatively explain 


\section{ADVANCED \\ Submitted to \\ MATERIALS}

the influence of the geometry on the resonance behavior. The strong field enhancement in this sub-wavelength plasmonic element promises applications in sensing and imaging, ${ }^{[36]}$ whilst the large energy dissipation by the electron oscillation on resonance suggests the implementation of perfect terahertz absorbers. ${ }^{[37]}$ The field confinement inside the cavity is suitable for terahertz integrated optics. Additionally, it is possible to realize negative-index materials from this terahertz coaxial microcavitiy array. ${ }^{[38]}$ A higher quality factor for the resonance can be obtained by replacing silicon with InSb.

\section{Experimental}

The silicon wafers were patterned using photolithography. Before spin coating the wafers with photoresist, the silicon wafers were thoroughly cleaned with acetone and isopropyl alcohol (IPA) followed by a dehydration bake. The cleaned wafer was then spin-coated with positive type photoresist (AZ 5214, MicroChemicals) and soft-baked on a hotplate. Using contact lithography both the mask and the wafer were brought in contact and exposed with UV light. The exposed wafer was developed using developer (AZ400K) to create openings for the etching. After developing the photoresist, the wafers were hard-baked to ensure the photoresist mask layer can withstand the selected etching process.

To obtain the coaxial microcavities, the photolithographically patterned silicon wafers were etched using the Bosch DRIE process. Bosch DRIE is a physical etching process to anisotropically etch silicon wafers and it can produce high aspect ratio vertical side walls. In the Bosch process, a mixture of $\mathrm{SF}_{6}$ and $\mathrm{C}_{4} \mathrm{~F}_{8}$ gas were alternatively permitted into the high vacuum chamber and ionized by applying RF power. The ionized $\mathrm{SF}_{6}$ etchant gas was accelerated towards the silicon wafer to create deep trenches in silicon wafer, $\mathrm{C}_{4} \mathrm{~F}_{8}$ gas mixture passivates the side walls to obtain smooth high aspect ratio vertical trenches. The etch rate of the silicon wafer using the Bosch DRIE process was approximately $2.5 \mu \mathrm{m} \mathrm{min}{ }^{-1}$ and 
Submitted to

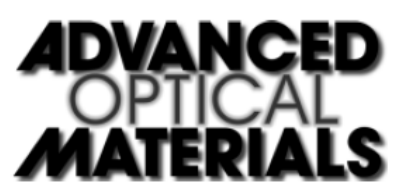

the samples were timed to etch $25 \mu \mathrm{m}$ and $50 \mu \mathrm{m}$ deep trenches into the silicon wafer. This

was confirmed using a profilometer (Ambios XP-2). On completion of etching, the photoresist was stripped off using acetone and the samples were cleaned using oxygen plasma to remove any polymer residue on the wafers.

\section{Acknowledgements}

W.W., M.B., and S.S. acknowledge Australian Postdoctoral Fellowships from the Australian Research Council (ARC) through Discovery Projects DP1095151, DP1092717, and DP110100262, respectively. C.F. acknowledges the ARC Future Fellowship funding scheme under FT100100585. W.W. and D.A. acknowledge funding via ARC Discovery Project DP120100200. S.S. acknowledges a Victoria Fellowship from Department of Business and Innovation, Government of Victoria. Technical assistance from Benjamin Ung, Jining Li, and Tiaoming Niu is gratefully acknowledged.

Received: ((will be filled in by the editorial staff))

Revised: ((will be filled in by the editorial staff)) Published online: ((will be filled in by the editorial staff))

_[1] Y. M. Tousi, V. Pourahmad, E. Afshari, Phys. Rev. Lett. 2012, 108, 234101.

_[2] K. Ishigaki, M. Shiraishi, S. Suzuki, M. Asada, N. Nishiyama, S. Arai, Electron. Lett.

2012, 48, 582 .

[3] K. Sengupta, A. Hajimiri, IEEE J. Solid-St. Circ. 2012, 47, 3013.

[4] B. M. Fischer, H. Helm, P. U. Jepsen, Proc. IEEE 2007, 95,1592.

[5] H. A. Atwater. Scientific Amer. 2007, 296, 56.

_[6] J. Saxler, J. Gómez Rivas, C. Janke, H. P. M. Pellemans, P. Haring Bolívar, H. Kurz, Phys. Rev. B 2004, 69, 155427.

[7] J. O’Hara, R. Averitt, A. J. Taylor, Opt. Express 2005, 13, 6117.

[8] T. I. Jeon, D. Grischkowsky, Appl. Phys. Lett. 2006, 88, 061113.

_[9] J. B. Pendry, L. Martín-Moreno, F. J. García-Vidal, Science 2004, 305, 847. 
_[10] C. R. Williams, S. R. Andrews, S. A. Maier, A. I. Fernández-Domínguez, L. MartínMoreno, F. J. García-Vidal, Nat. Photon. 2008, 2, 175.

_[11] C. R. Williams, M. Misra, S. R. Andrews, S. A. Maier, S. Carretero-Palacios, S. G. Rodrigo, F. J. García-Vidal, L. Martin-Moreno, Appl. Phys. Lett. 2010, 96, 011101.

_[12] M. Navarro-Cía, M. Beruete, M. Sorolla, S. A. Maier, Plasmonics 2011, 6, 295.

_[13] J. Gómez Rivas, M. Kuttge, P. Haring Bolívar, H. Kurz, J. A. Sánchez-Gil, Phys. Rev. Lett. 2004, 93, 256804.

_[14] J. Gómez Rivas, M. Kuttge, H. Kurz, P. Haring Bolívar, J. A. Sánchez-Gil, Appl. Phys. Lett. 2006, 88, 082106.

[15] W. A. Murray, W. L. Barnes, Adv. Mater. 2007, 19, 3771.

_[16] T. H. Isaac, W. L. Barnes, E. Hendry, Appl. Phys. Lett. 2008, 93, 241115.

_[17] V. Giannini, A. Berrier, S. A. Maier, J. A. Sánchez-Gil, J. Gómez Rivas, Opt. Express 2010, 18, 2797.

_[18] A. Berrier, R. Ulbricht, M. Bonn, J. Gómez Rivas, Opt. Express 2010, 18, 23226.

_[19] S. M. Hanham, A. I. Fernández-Domínguez, J. H. Teng, S. S. Ang, K. P. Lim, S. F. Yoon, C. Y. Ngo, N. Klein, J. B. Pendry, S. A. Maier, Adv. Mater. 2012, 24, OP226.

_[20] A. Berrier, P. Albella, M. Ameen Poyli, R. Ulbricht, M. Bonn, J. Aizpurua, J. Gómez Rivas, Opt. Express 2012, 20, 5052.

_[21] J. Salvi, M. Roussey, F. I. Baida, M.-P. Bernal, A. Mussot, T. Sylvestre, H. Maillotte, D. van Labeke, A. Perentes, I. Utke, C. Sandu, P. Hoffmann, B. Dwir, Opt. Lett. 2005, 30, 1611.

_[22] M. I. Haftel, C. Schlockermann, G. Blumberg, Appl. Phys. Lett. 2006, 88, 193104.

[23] M. I. Haftel, C. Schlockermann, G. Blumberg, Phys. Rev. B 2006, 74, 235405.

[24] S. M. Orbons, A. Roberts, D. N. Jamieson, M. I. Haftel, C. Schlockermann, D.

Freeman, B. Luther-Davies, Appl. Phys. Lett. 2007, 90, 251107. 
Submitted to

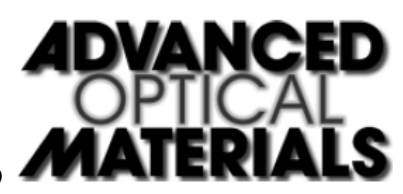

[25] J. Pritz, L. M. Woods, Solid St. Comm. 2008, 146, 345.

_[26] E. Jan, R. Vesseur, F. Javier García de Abajo, A. Polman, Nano Lett. 2009, 9, 3147.

_[27] J. Zhang, J.-Y. Ou, N. Papasimakis, Y. Chen, K. F. MacDonald, N. I. Zheludev, Opt. Express 2011, 19, 23279.

[28] D. Hashimshony, I. Geltner, G. Cohen, Y. Avitzour, A. Zigler, C. Smith, J. Appl. Phys. 2001, 90, 5778 .

[29] G. Baccarani, Solid St. Electron. 1975, 18, 579.

_[30] P. U. Jepsen, B. M. Fischer, Opt. Lett. 2005, 30, 29.

[31] R. D. Kekatpure, A. C. Hryciw, E. S. Barnard, M. L. Brongersma, Opt. Express 2009, $17,24112$.

[32] J. Zhang, J.-Y. Ou, K. F. MacDonald, N. I. Zheludev, J. Opt. 2012, 14, 114002.

[33] S. A. Maier. Plasmonics: Fundamentals and Applications, Springer, 2007.

[34] P. B. Catrysse, S. Fan, Appl. Phys. Lett. 2009, 94, 231111.

[35] D. M. Pozar, Microwave Engineering, John Wiley \& Sons, 2009.

[36] A. Weber-Bargioni, A. Schwartzberg, M. Cornaglia, A. Ismach, J. J. Urban, Y.-J.

Pang, R. Gordon, J. Bokor, M. B. Salmeron, D. F. Ogletree, P. Ashby, S. Cabrini, P. J.

Schuck, Nano Lett. 2011, 11, 1201.

_[37] C. M. Watts, X. Liu, W. J. Padilla, Adv. Mater. 2012, 24, OP98.

[38] S. P. Burgos, R. de Waele, A. Polman, H. A. Atwater, Nat. Mater. 2010, 9, 407. 


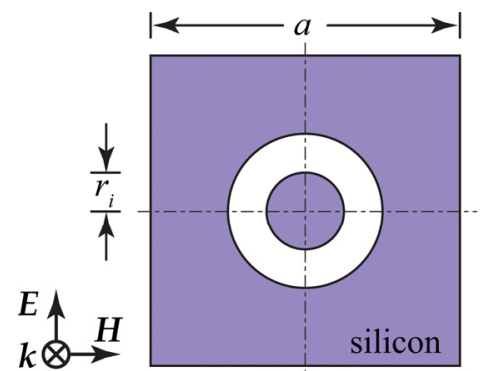

(a)

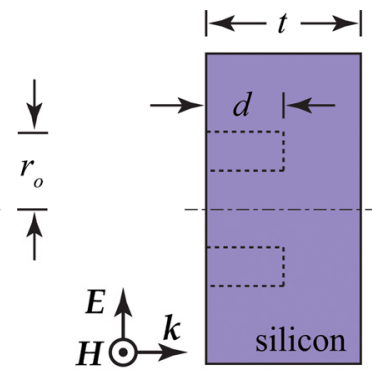

(b)

Figure 1. Diagram for a unit cell of the coaxial microcavity in a silicon substrate. (a) Top view, and (b) side view. The square unit cell size $a$ is fixed at $200 \mu \mathrm{m}$, and the silicon substrate thickness $t$ equals $270 \mu \mathrm{m}$. The inner radius $\left(r_{i}\right)$, outer radius $\left(r_{o}\right)$, and cavity depth (d) are given in Table 1 . The diagram is not to scale.
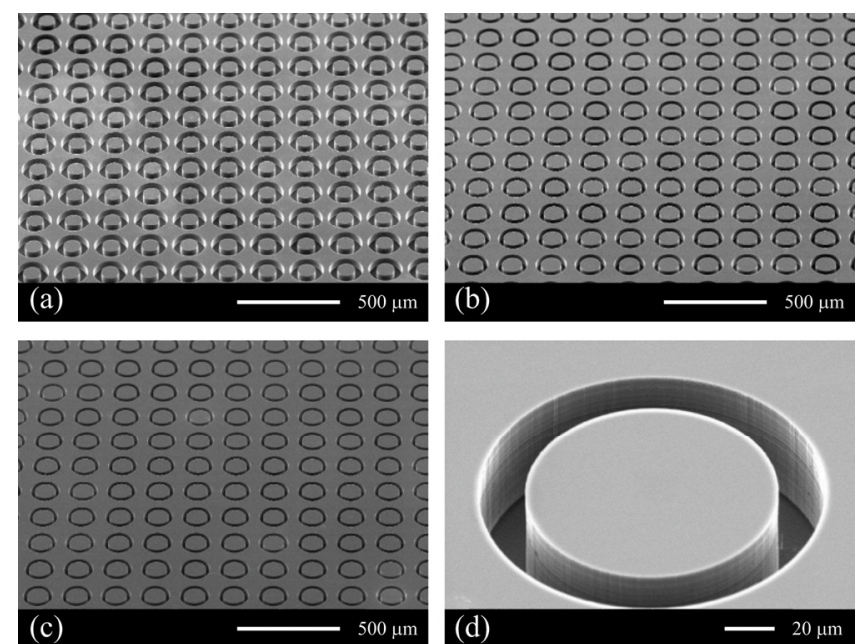

Figure 2. Scanning electron micrographs of the fabricated microcavities. Partial view of the cavity arrays (a) L40, (b) L30, and (c) L20. (d) Magnified view of a single cavity L20. The micrographs are taken at the tilt angle of $45^{\circ}$. All of the samples shown here have a depth of $50 \mu \mathrm{m}$.
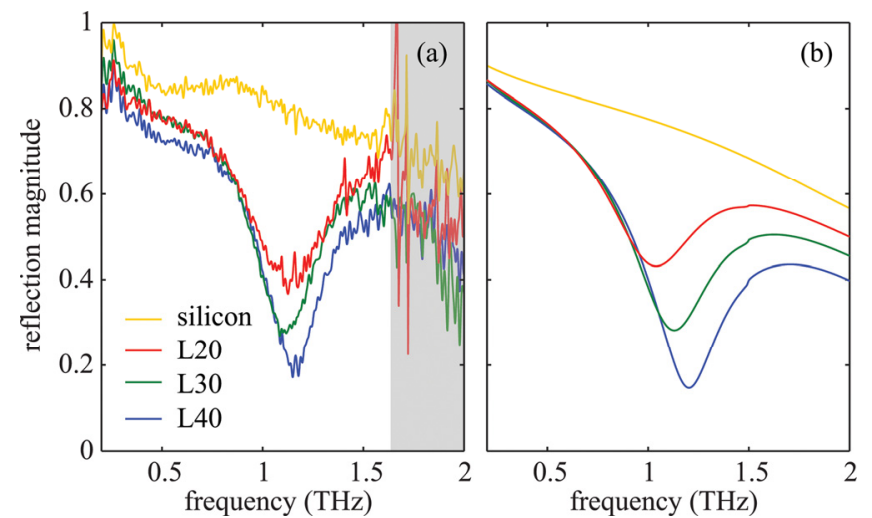

Figure 3. Normalized reflection spectra for microcavity arrays with $50 \mu \mathrm{m}$ cavity depth. (a) Experimental and (b) simulation results. The reflection spectrum for the bare silicon is included for comparison. All the spectra are normalized to the reference taken from the goldcoated silicon wafer. The shaded area in (a) marks the limit of the system dynamic range. ${ }^{[30]}$ 


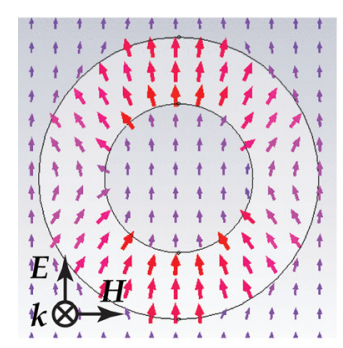

(a)

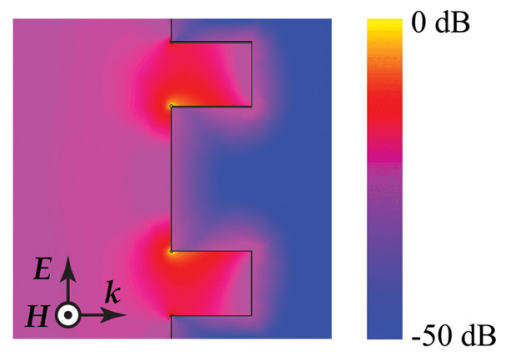

(b)

Figure 4. Numerically resolved field distribution in the coaxial microcavity. (a) Instantaneous electric field pattern over the opening of the coaxial cavity, and (b) absolute electric field strength across the center of the cavity. The cavity dimensions are according to sample L40. The field plots are on resonance at $1.2 \mathrm{THz}$. The maximum field strength is set to $0 \mathrm{~dB}$.

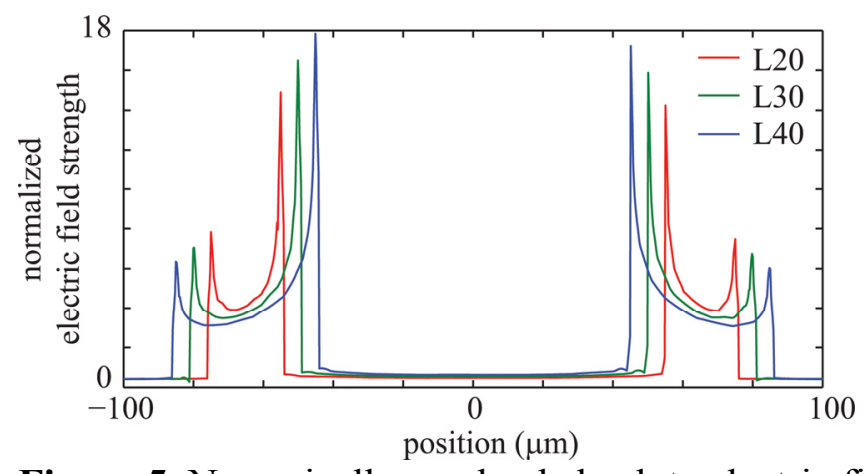

Figure 5. Numerically resolved absolute electric field strength for the $50 \mu \mathrm{m}$ cavities. The frequency for each field profile is at the resonance of the structure, i.e. 1.04, 1.13, and 1.20 THz for samples L20, L30, and L40, respectively. The field profile is located across the opening of the cavity along the incident polarization. The field strength is the component normal to the cavity walls, and is normalized to that on the bare silicon surface.

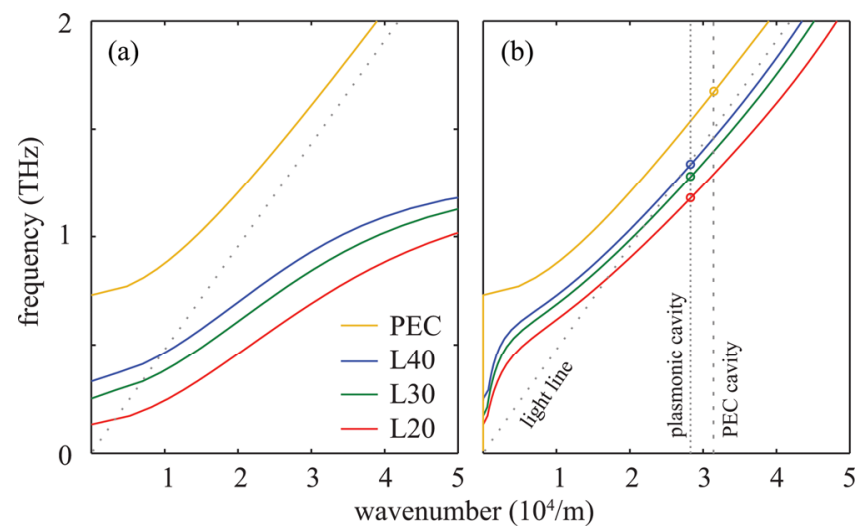

Figure 6. Dispersion diagram for the TE 11 mode in the PEC and plasmonic coaxial waveguides. (a) Lossless plasmonic cavities, i.e. $\Im(E c)=0$, and (b) lossy plasmonic cavities. The dispersion for the PEC waveguide depends only on the average radius, ${ }^{[35]}$ set to $65 \mu \mathrm{m}$ as that for all the plasmonic cavity designs. The dispersion for the plasmonic waveguides is determined from Equation 1. The vertical lines indicate the wavenumber $\beta_{0}$ where the fundamental resonance is expected for cavities with a depth of $50 \mu \mathrm{m}$. 

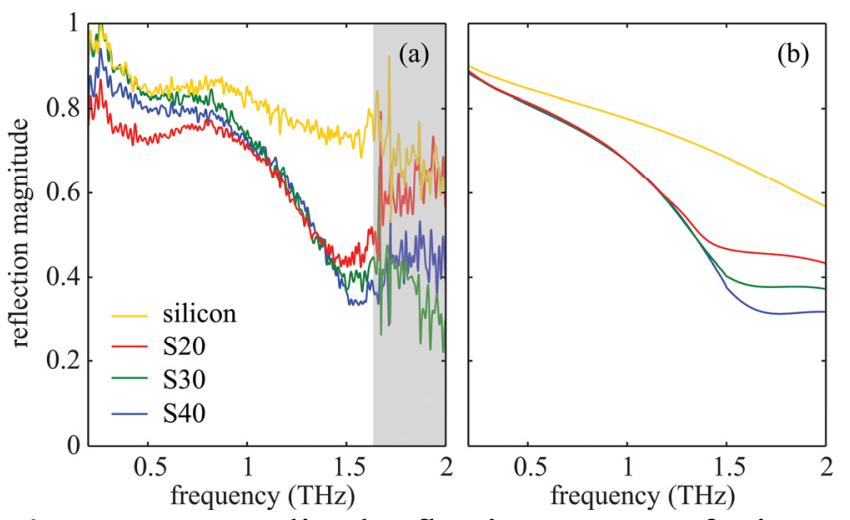

Submitted to

Figure 7. Normalized reflection spectra of microcavity arrays with $25 \mu \mathrm{m}$ cavity depth. (a) Experimental and (b) simulation results. The reflection spectrum for the bare silicon is included for comparison. All the spectra are normalized to the reference taken from the goldcoated silicon wafer. The shaded area in (a) marks the limit of the system dynamic range.

Table 1. Dimensions of the fabricated coaxial cavities. All the units are in micrometers. The average radius $r_{\mathrm{avg}}$ equals $65 \mu \mathrm{m}$ for all of the samples.

\begin{tabular}{ccccc}
\hline Sample & $\begin{array}{c}\text { Cavity } \\
\text { depth } d\end{array}$ & $\begin{array}{c}\text { Wall gap } \\
g=r_{0}-r_{i}\end{array}$ & $\begin{array}{c}\text { Outer } \\
\text { radius } r_{0}\end{array}$ & $\begin{array}{c}\text { Inner } \\
\text { radius } r_{i}\end{array}$ \\
\hline L40 & 50 & 40 & 85 & 45 \\
L30 & 50 & 30 & 80 & 50 \\
L20 & 50 & 20 & 75 & 55 \\
S40 & 25 & 40 & 85 & 45 \\
S30 & 25 & 30 & 80 & 50 \\
S20 & 25 & 20 & 75 & 55 \\
\hline
\end{tabular}




\section{Submitted to

The table of contents entry should be fifty to sixty words long, written in the present tense, and refer to the chosen figure.

\section{Table of Contents Entry}

Coaxial microcavities etched into the surface of a doped silicon substrate are shown to support localized surface plasmon resonances (LSPRs) at terahertz frequencies. This study is a promising pathway for development of terahertz devices with applications in the areas of photonic integrated circuits, molecular sensing, and subwavelength imaging.

\section{Keywords}

plasmonics, localized surface plasmon resonance, terahertz, metamaterial, coaxial cavity

\section{Authors}

Withawat Withayachumnankul, ${ }^{*}$ Charan Manish Shah, Christophe Fumeaux, Korbinian Kaltenecker, Markus Walther, Bernd M. Fischer, Derek Abbott, Madhu Bhaskaran, and Sharath Sriram*

\section{Title}

Terahertz Localized Surface Plasmon Resonances in Coaxial Microcavities

\section{ToC Figure}
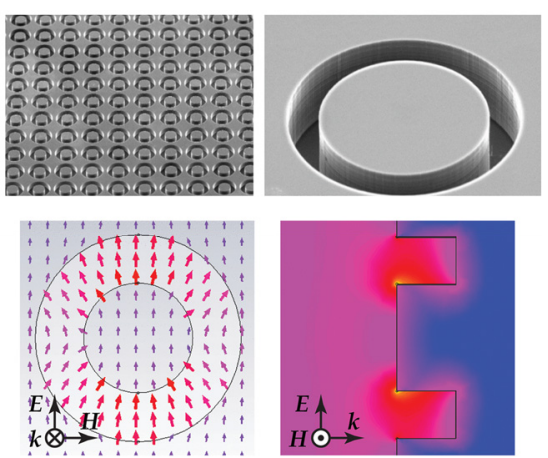